\title{
Role of grape seed polyphenols in Alzheimer's disease neuropathology
}

\author{
This article was published in the following Dove Press journal: \\ Nutrition and Dietary Supplements \\ 14 August 2010 \\ Number of times this article has been viewed
}

\author{
Giulio Maria Pasinetti ${ }^{1,2}$ \\ Lap Ho' \\ 'Department of Neurology, \\ Mount Sinai School of Medicine, \\ New York; ${ }^{2}$ Geriatric Research, \\ Education and Clinical Center, \\ James J Peters Veteran Affairs \\ Medical Center, Bronx, \\ New York, USA
}

Correspondence: Giulio Maria Pasinetti Mount Sinai School of Medicine, I Gustave L Levy Place, Box I I37, New York, NY 10029, USA

Tel + I 2122417938

Fax + I 2128769042

Email giulio.pasinetti@mssm.edu
Abstract: Alzheimer's disease (AD) is an age-related neurodegenerative condition characterized by a progressive decline in cognitive function. $\mathrm{AD}$ affects approximately five million people in the US, creating a devastating financial burden on health care costs and an emotional burden on caregivers. To date, there is no cure for $\mathrm{AD}$, so researchers are continually exploring novel avenues for the prevention and treatment of this condition. In this article, we present some findings from our laboratory and those of others on the potential benefits of a grape seed polyphenolic extract (GSPE) for the prevention and treatment of $\mathrm{AD}$, including its chemical composition, bioactivity, bioavailability, safety, and tolerability, and the mechanisms by which it interferes with AD pathogenesis. Findings presented in this review article support the development of GSPE as a preventative and/or therapeutic agent in AD.

Keywords: alzheimer's disease, neurodegeneration, polyphenols

\section{Introduction}

Alzheimer's disease (AD) is the most common type of dementia in the US. Victims of AD commonly display a loss of memory, inability to learn new things, loss of language function, deranged perception of space, inability to do calculations, depression, delusions, and other cognitive deficiencies. AD is ultimately fatal within five to 10 years of its onset. It is estimated that approximately five million people in the US currently have $\mathrm{AD},{ }^{1}$ with an estimated cost to society of more than $\$ 100$ billion per year. Up to 14 million people in the US are projected to be affected by AD by the middle of this century if effective therapies are not developed. ${ }^{1}$ While there is currently no cure for AD, even delaying AD onset by a few years would lead to significant reductions in disease prevalence and, consequently, its burden on health care systems. The few agents that are approved by the US Food and Drug Administration for the treatment of AD have only modest efficacy in modifying clinical symptoms, and none appear to affect disease progression or prevention (reviewed in). ${ }^{2}$ Historically, the most candidate therapeutics have been directed toward cholinergic neurons, which are especially vulnerable in AD and cognitive alterations (reviewed in). ${ }^{2,3}$ More recently, agents are being developed to interfere with the $\beta$-amyloid ( $\mathrm{A} \beta$ ) protein precursor pathway, which is thought to be responsible for $\mathrm{A} \beta$-mediated neuronal dysfunction and death. ${ }^{4,5}$

\section{Neuropathologic features of Alzheimer's disease}

While the classification and diagnosis of $\mathrm{AD}$ is based on the cognitive behavior of an individual, the roots of the disease lie in the neurologic pathology of its victims. ${ }^{6}$ 
The two defining neuropathologic features of AD are abnormal aggregation and deposition of $A \beta$ peptides and tau protein in the brain as, respectively, extracellular neuritic plaque and intracellular neurofibrillary tangles (NFT). ${ }^{7}$

A $\beta$ species with different amino and carboxyl termini are generated from the ubiquitously expressed amyloid precursor protein through sequential proteolysis by $\beta$ - and "pro-amyloidogenic" $\gamma$-secretases. ${ }^{4,5,8}$ A 40-amino acid form of $A \beta\left(A \beta_{1-40}\right)$ is the major secreted species. However, a 42 -amino acid form of $A \beta\left(A \beta_{1-42}\right)$, which contains two additional residues at its carboxyl terminus, is thought to initiate AD pathogenesis. ${ }^{9} \mathrm{~A} \beta_{1-42}$ aggregates much more readily than $\mathrm{A} \beta_{1-40}$ in vitro, and is also deposited earlier and more consistently in $\mathrm{AD}$ brain parenchyma. In humans, AD-causing mutations elevate plasma $A \beta_{1-42}$ levels by approximately $30 \%-100 \%{ }^{10}$ Even mutations showing small increases in $\mathrm{A} \beta_{1-42}$ levels are associated with the onset of dementia in the fourth or fifth decade of life. In the Tg2576 transgenic mouse model of $\mathrm{AD}$, the same mutations produce increases in $\mathrm{A} \beta_{1-42}$ levels and markedly accelerate $\mathrm{A} \beta$ deposition. ${ }^{11}$ Based on this evidence, a major effort from both academia and industry is presently focused on developing pharmacologic strategies that would delay the initiation and/or slow the progression of AD-type $\mathrm{A} \beta$ neuropathology in the brain. Recent evidence from experimental AD mouse models indicates that accumulation of soluble high molecular weight (HMW) oligomeric $\mathrm{A} \beta$ species in the brain, rather than deposition of neuritic plaque per se, may be specifically related to spatial memory reference deficits. In particular, experimental evidence demonstrated that HMW oligomeric A $\beta$ species may disrupt hippocampal long-term potentiation and synaptic plasticity, and induce synaptic deficits. ${ }^{12-15}$ Consistent with the hypothesis that soluble HMW oligomeric A $\beta$ species play a key role in AD memory dysfunction, experimental therapeutic evidence demonstrated that neutralization of soluble HMW A $\beta$ oligomeric species in the brain causally improves spatial memory functions in a mouse model of AD. ${ }^{16}$

Despite strong genetic data arguing that $\mathrm{A} \beta$ neuropathology is sufficient to cause $\mathrm{AD},{ }^{17}$ progressive cognitive decline and neuron and synapse loss in AD are best correlated with tau neuropathology. ${ }^{18}$ In the AD brain, tau proteins, particularly hyperphosphorylated tau, are found aggregated into progressively larger polymeric species that are ultimately deposited as insoluble NFT. ${ }^{19}$ NFTs themselves are not necessarily the tau species inducing neurotoxicity, because evidence in experimental mouse models suggests that neuronal loss and memory impairment may occur before NFT formation in the brain. ${ }^{20,21}$ Instead, evidence indicates that accumulation of multimeric tau aggregates may play a more important role in $\mathrm{AD}$ memory dysfunction. ${ }^{21}$ Consistent with this hypothesis, in transgenic mouse models, levels of tau multimers are significantly correlated with memory index. ${ }^{21}$

Moreover, neuronal loss and memory impairment in a mouse model of tauopathy can be mitigated by reducing tau expression without affecting the number of NFTs. ${ }^{20}$ Aggregation of tau is a seed nucleation process. Formations of hyperphosphorylated oligomers serve as nucleation sites that sequester additional hyperphosphorylated tau as well as normal nonphosphorylated tau. ${ }^{22}$ Thus, a predominant theory of tau-mediated neurodegeneration is based on a "toxic gain of function" model, in which abnormally phosphorylated tau promotes sequestration of both hyperphosphorylated and normal tau from microtubules, leading to microtubule instability and alteration of microtubule-mediated processes, including abnormalities in axon transport among others. ${ }^{22}$

\section{Oligomeric $A \beta$ and tau species in the brain as a target for AD therapy}

These considerations strongly suggest that reducing the accumulation of soluble oligomeric $A \beta$ peptides and tau species in the brain, as opposed to dissociating or preventing NP and/or NFT formation or deposition, may be a more productive approach to AD therapy. In light of this, our laboratory and others have recently investigated polyphenolic compounds from a specific grape seed polyphenolic extract (GSPE), and have demonstrated its ability to interfere with the aberrant aggregation of $\mathrm{A} \beta$ and tau, ${ }^{23,24}$ exert bioactivity in vivo, improve cognitive function, and reduce brain neuropathology in experimental AD models. ${ }^{25}$

\section{Potential health benefits of grape seed polyphenols}

Grape seeds are a rich source of polyphenolic compounds, with proanthocyanidins being the primary grape seed polyphenolic constituents. Grape seed polyphenols have been reported to possess a broad spectrum of pharmacologic and medicinal properties, such as mitigating breast cell carcinogenesis, ${ }^{26}$ modulating blood pressure in individuals with prehypertension, ${ }^{27}$ maintaining glucose homeostasis in diabetic conditions, ${ }^{28}$ protecting against ischemia-related injuries, ${ }^{29}$ promoting dermal wound healing, ${ }^{30}$ and protecting against diabetic nephropathy. ${ }^{31}$ Grape seed polyphenols (as well as most other polyphenolic compounds) are potent antioxidants. Indeed, the health benefits of polyphenolic compounds, including grape seed proanthocyanidins, have traditionally 
been attributed to their antioxidant activities. ${ }^{32}$ For example, antioxidant activities might be a major contributory factor to the role of grape seed polyphenols in protection against breast cancer, ${ }^{26}$ wound healing, ${ }^{30}$ and ischemic injuries. ${ }^{29}$ However, grape seed polyphenols may induce additional beneficial disease-modifying mechanisms. For example, grape seed polyphenols might mitigate diabetic nephropathy by mechanisms involving reduced expression of advanced glycation end products and connective tissue growth factor in the kidney. ${ }^{31}$ In another example, grape seed polyphenols might promote the maintenance of glucose homeostasis in diabetic conditions, in part, by activating insulin receptor signaling pathways. ${ }^{33}$ Recent observations from in vitro experimental studies ${ }^{24,25}$ and preclinical studies ${ }^{34}$ demonstrated that grape seed polyphenols may interfere with specific neuropathogenic mechanisms underlying $\mathrm{AD}$ and suggest a potential novel role of grape seed polyphenols for treating AD.

\section{Chemical composition}

The GSPE is a highly purified extract from grape seed (Vitis vinifera, grape seed extract-GSE, Polyphenolics, Madera, CA). It is characterized by a high degree of purity as determined by the amount of the total phenolic compound greater than about $90 \%$ by weight, as determined by the Folin-Ciocalteu method. ${ }^{35}$ The GSPE has a high amount of low molecular weight phenolic compounds (about $50 \%$ by weight), wherein low molecular weight phenolic compounds are monomers, dimers, trimers, tetramers, and pentamers. High pressure liquid chromatographic analysis revealed that the GSPE is comprised of catechin and epicatechin in monomeric and polymeric forms. ${ }^{25}$

\section{Bioavailability}

We explored the bioavailability of GSPE polyphenolic components in vivo. In initial preclinical studies using the Sprague Dawley rat model, we measured plasma pharmacokinetic responses ( $0-8$ hours) to GSPE and their methylated and glucuronidated metabolites in the blood following oral administration. Dimer, trimer, or larger oligomeric GSPE proanthocyanidins were not detected in plasma following acute administration. ${ }^{36}$ Interestingly, however, we found that repeated exposure to GSPE led to accumulations of higher catechin, epicatechin, gallic acid, and their metabolites in the blood. ${ }^{36}$ In the brain, we found detectable contents of catechin, epicatechin, and their respective metabolites following acute oral administration of the GSPE. Consistent with our observation in blood, repeated oral administration of the GSPE led to accumulation of higher concentrations of catechin, epicatechin, and their metabolites in the brain. ${ }^{36}$

\section{GSPE interferes with A $\beta$-mediated neuropathology and cognitive deterioration}

We found that GSPE potently inhibits the aggregation of $A \beta$ peptides into neurotoxic soluble $A \beta$ oligomeric species independently using a gel motility retardation assay and a photo-induced crosslinking of unmodified protein assay. ${ }^{23,25}$ Moreover, we demonstrated that the GSPE blocks $A \beta$ fibril formation by interfering with protofibril formation, preprotofibrilar oligomerization, and initial coil to $\alpha$-helix/ $\beta$-sheet secondary structure transitions. ${ }^{23}$ Lastly, we established that all monomeric, multimeric, and polymeric GSPE isolates exert anti-A $\beta$ oligomerization activity in vitro and potently inhibit initial peptide oligomerization of both $\mathrm{A} \beta_{1-40}$ and $\mathrm{A} \beta_{1-42}$ (unpublished observation).

Based on evidence for the bioavailability of GSPE and its efficacy in interfering with mechanisms relating to $A \beta$ aggregation, we continue to explore the potential role of GSPE to modulate $A \beta$-related phenotypes in the $\operatorname{Tg} 2576$ transgenic mouse model of Alzheimer's disease. Tg2576 mice are engineered to express human $A \beta$ peptides that are responsible for age-related induction of Alzheimer's disease-type amyloid neuropathology and cognitive dysfunction. ${ }^{37} \mathrm{We}$ found that dietary supplementation with the GSPE $(200 \mathrm{mg} / \mathrm{kg}$ body weight, equivalent to human consumption of $1 \mathrm{~g}$ polyphenols per day) is well tolerated in Tg2576 mice. ${ }^{25}$ Moreover, we found that dietary supplementation with the GSPE, starting at six months of age, attenuated the development of AD-type cognitive deterioration coincidental with reduced HMW soluble oligomeric $A \beta$ in the brain when animals were assessed at 10 months of age. ${ }^{25} \mathrm{In}$ an independent series of preclinical studies, Wang et $\mathrm{al}^{34}$ also reported that dietary supplementation with GSPE significantly attenuated the development of $A \beta$ neuropathology and neuroinflammation in the brain of another AD mouse model (double transgenic APPswe/PSIdE9 mice). Thus, observations from independent laboratories using different $\mathrm{AD}$ mouse models confirmed the preclinical efficacy of GSPE to mitigate AD-type A $\beta$ neuropathology.

Consistent with our observation that only monomeric catechin, epicatechin, and gallic acid are bioavailable in the brain, ${ }^{36}$ we recently found that a GSPE preparation enriched for the monomeric components is effective in reducing $A \beta$ neuropathology and promoting cognitive function in Tg2576 mice (unpublished observation). In contrast, 
a GSPE preparation enriched for the polymeric components is not capable of modulating $A \beta$-related neuropathologic or cognitive dysfunctions in $\mathrm{Tg} 2576$ mice (unpublished observation).

\section{Potential role of GSPE in mitigating tau-mediated neuropathologic phenotypes}

In many respects, the modeling of tau paired helical filaments (PHFs) parallels the modeling of amyloid fibrils from A $\beta$ peptide fragments. ${ }^{38}$ Amyloid fibrils have long been characterized by $\beta$-sheet formation and, recently, by parallel superpleated $\beta$-structures. ${ }^{39}$ In contrast with the hydrophobic character and a strong propensity for $A \beta$ peptides, normal tau is a highly soluble hydrophilic protein in a random coil conformation without evidence of $\beta$-structure. ${ }^{40}$ It is not surprising that the presence of cross- $\beta$ conformation in PHFs observed in several neurodegenerative disorders characterized by abnormal tau aggregation was initially controversial. ${ }^{41}$ Nevertheless, recent studies of PHFs isolated from AD brains, in addition to synthetic PHFs assembled from tau constructs, strongly suggest a predominant cross- $\beta$ conformation of tau proteins in these filamentous structures and that a transition from random coils to cross- $\beta$ structures is a driving force of tau aggregation. ${ }^{42-44}$ In some studies, an $\alpha$-helical structure has also been detected. ${ }^{45}$ The presence of predominant cross- $\beta$ conformation in PHFs suggests that fibrillogenesis of tau may be similar to that in other amyloids, such as $A \beta$. Based on this and on our recent evidence that GSPE inhibits the aggregation of $A \beta$ peptides and prevents $A \beta$-mediated neuropathology and cognitive deterioration in the Tg2576 AD mouse model, ${ }^{25}$ we hypothesized that GSPE might similarly interfere with the generation of neuropathologic tau fibrils.

Formation of tau into a $\beta$-sheet structure has been attributed to the short motifs ${ }^{275} \mathrm{VQIINK}^{280}$ and ${ }^{306} \mathrm{VQIVYK}^{311}$ in the second and third repeat of the microtubule-binding domain, respectively. ${ }^{38,44} \mathrm{X}$-ray diffraction patterns indicate that $\beta$-chains run nearly normal (perpendicular to the fiber directions) in a cross- $\beta$ arrangement. Aggregations of $A \beta$ peptides and tau are nucleation processes. The smallest peptide that can still form filaments has been identified as ${ }^{309} \mathrm{VYK}^{311}$, which has been proposed to initiate nucleation in vivo. ${ }^{46}$ Based on this, we used spontaneous aggregations of a synthetic Ac- ${ }^{306}$ VQIVYK ${ }^{311}$ tau peptide containing the nucleation site sequence as an in vitro model system in our feasibility studies to explore the potential efficacy of the GSPE to modulate tau aggregation. We found that the GSPE might interfere with the formation of tau aggregates and the stability of those that are formed. In particular, we found that coincubation of the synthetic tau peptide with GSPE inhibits the formation of tau aggregates in a dose-dependent manner. ${ }^{24}$ Moreover, the presence of low concentrations of the GSPE corresponding to a molar ratio of 1:10 GSPE relative to tau peptide was effective in significantly reducing aggregations of the tau peptide. ${ }^{24}$

We further found that the GSPE might also benefit taumediated neuropathologic responses by interfering with the stability of neurotoxic tau fibrils. In particular, we observed significant dissociation of preformed tau peptide aggregates when these are incubated with the GSPE at a molar ratio of 1:1 GSPE relative to tau peptide. ${ }^{24}$ Collectively, our evidence suggests that GSPE may reduce the accumulation of neurotoxic tau fibrils in the brain. In ongoing studies exploring the potential in vivo efficacy of GSPE in mitigating tau-mediated neurodegenerative phenotypes, we found that dietary supplementation with GSPE $175 \mathrm{mg} / \mathrm{kg}$ body weight per day (equivalent to human consumption of $900 \mathrm{mg}$ polyphenols per day) may also reduce tau neuropathology in an experimental mutant tau mouse model (unpublished observation). Ongoing studies are exploring whether the GSPE might attenuate the development of tau-related cognitive dysfunctions in transgenic mutant tau mouse models.

\section{Tolerability and safety of GSPE}

The GSPE is highly tolerable and safe in experimental animal models. For example, we found that five months of treatment with GSPE $200 \mathrm{mg} / \mathrm{kg} /$ day in a Tg2576 mouse model of $\mathrm{AD}$ amyloid neuropathology was very safe, with no detectable adverse events. ${ }^{25}$ Moreover, treatment of rats with GSPE doses up to $17,500 \mathrm{mg} / \mathrm{kg} /$ day was also found to be very safe and tolerable. ${ }^{47}$ Translating GSPE treatments from animal models to human dosage using the formulation recommended by the Food and Drug Administration for converting equivalent drug dosage between species (http://www.fda.gov/cber/gdlns/dose. $\mathrm{htm}$ ), we calculated that a dosage of $200 \mathrm{mg} / \mathrm{kg} /$ day in mice and $17,500 \mathrm{mg} / \mathrm{kg} /$ day in rats is equivalent to a human dosage of $1000 \mathrm{mg} /$ day and 20,000 mg/day, respectively. In humans, GSPE at $300 \mathrm{mg} /$ day for eight weeks was reported to be highly safe and tolerable in a clinical study exploring the role of the GSPE in subjects with prehypertensive conditions meeting the JNC 7 criteria (systolic blood pressures 120-139 $\mathrm{mmHg}$ and diastolic blood pressures $80-89 \mathrm{mmHg}){ }^{27}$ In particular, the study reported that treatment with GSPE $300 \mathrm{mg} /$ day significantly reduced both systolic and diastolic blood pressure by an average of $8 \mathrm{mmHg}$ and $5 \mathrm{mmHg}$, respectively, and no adverse events were observed in response to GSPE treatment. ${ }^{27}$ 
Collectively, this evidence suggests that GSPE is likely to be highly safe and tolerable for treatment in humans. However, it is necessary to conduct more detailed safety and efficacy studies in AD patients.

Evidence demonstrated that dietary supplementation with GSPE $200^{25}$ and 175 (unpublished observation) mg/ $\mathrm{kg}$ body weight per day significantly mitigated the development of, respectively, $A \beta$ - and tau-neuropathologic phenotypes in transgenic mouse models. Translating to human dosages, 200 and $175 \mathrm{mg} / \mathrm{kg}$ body weight per day is equivalent to daily consumption of, respectively, $1 \mathrm{gm}$ and $900 \mathrm{mg}$ per day. Ongoing preclinical studies are continuing to explore whether lower doses of GSPE might be efficacious in mitigating $\mathrm{A} \beta$ - and/or tau-mediated responses in vivo and the pharmacokinetics of blood GSPE polyphenolic components that are associated with therapeutic benefits in transgenic animal models of $A \beta$ and tau pathology. Information gathered will provide the logical basis for selecting the most appropriate human doses for the development of clinical trials to test the safety and efficacy of the GSPE in AD.

\section{Summary}

Recent in vitro studies demonstrate that GSPE interferes with the aggregation of $A \beta$ peptides and tau into neurotoxic oligomeric $A \beta$ aggregates and tau fibril conformers (Figure 1, panel A). Moreover, our evidence suggests that the GSPE may also destabilize preformed A $\beta$ and tau aggregates (Figure 1, panel B). Thus, GSPE might modulate $\mathrm{AD}$ dementia by beneficially modulating both $\mathrm{A} \beta$ and taumediated neuropathologic mechanisms (Figure 1, panels A and B). More importantly, our in vivo preclinical studies

A

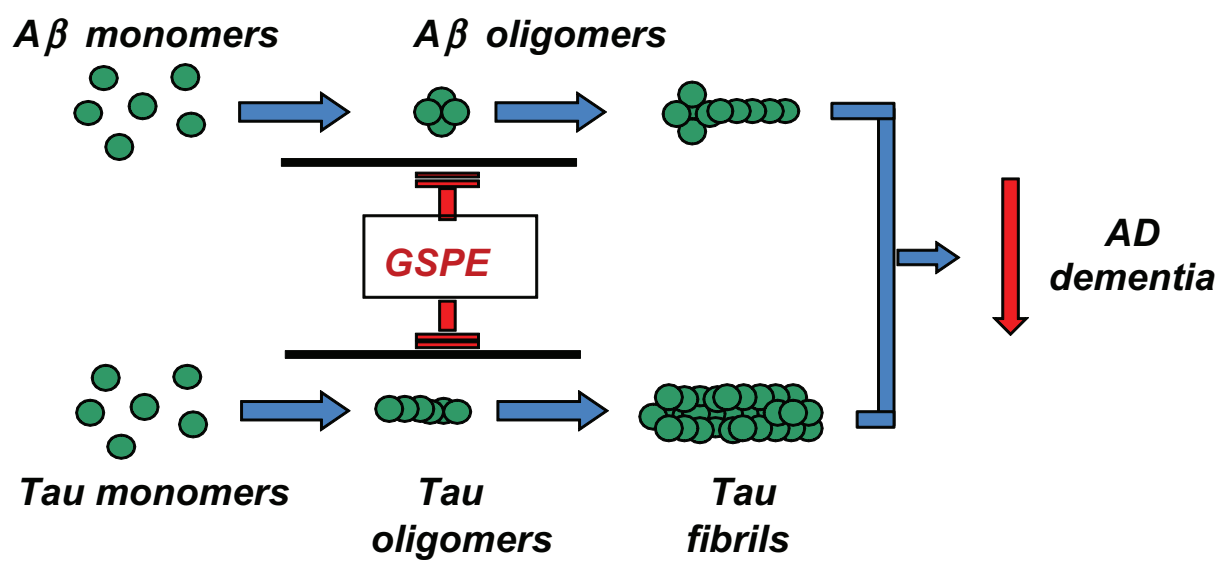

B

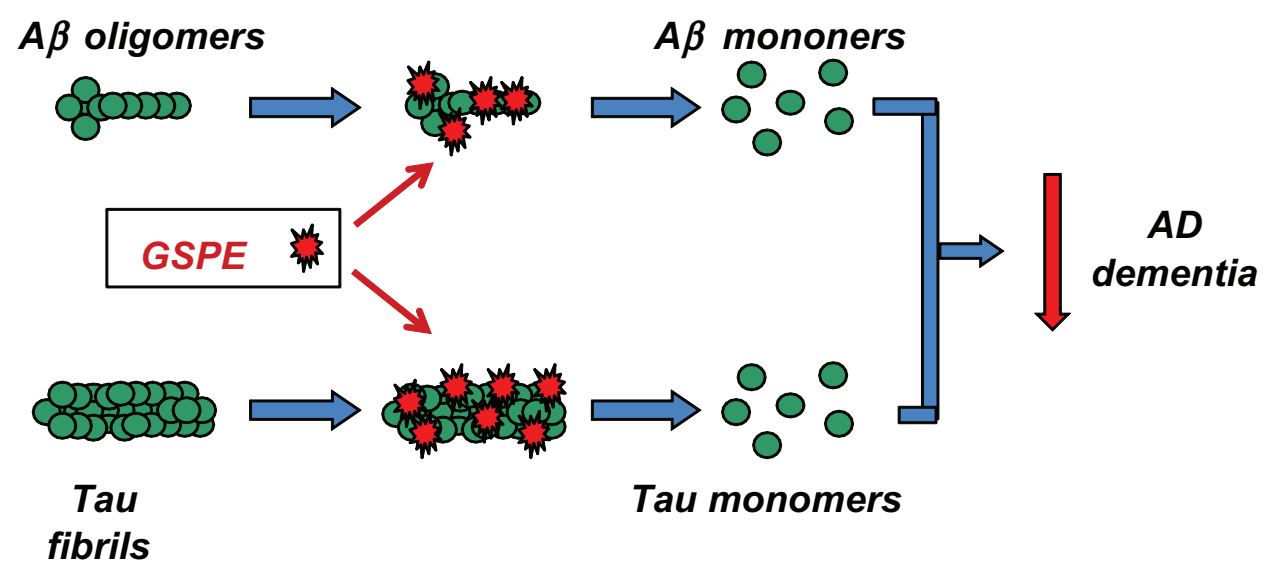

Figure I GSPE might benefit AD by simultaneously interfering with the generation and stability of neurotoxic A $\beta$ and tau oligomeric conformers. A) GSPE interferes with protein-protein interactions necessary for the assembly of $A \beta$ peptides or tau proteins into neurotoxic oligomeric aggregates. B) GSPE may intercalate into preformed $A \beta$ or tau oligomeric aggregates, which destabilizes the normally tight ultrastructure and leads to the dissociation of $A \beta$ aggregates and tau fibrils. Abbreviations: AD, Alzheimer's disease; GSPE, grape seed polyphenolic extract. 
support the efficacy of GSPE to mitigate A $\beta$ - or mutant tau-mediated neuropathologic phenotypes. Thus, GSPE might benefit $\mathrm{AD}$ by simultaneously interfering with the two hallmark neuropathologies of the disease. These new observations of the effects of GSPE treatment on AD pathologic features, taken together with the demonstrated bioavailability as well as safety and tolerability, strongly support the continued development of GSPE for AD prevention and therapy.

\section{Acknowledgement}

This work was supported by a National Institutes of Health grant PO1AT004511 to GMP. GMP and LH are named inventors of a pending patent application filed by Mount Sinai School of Medicine for grape seed polyphenolic extract. In the event that the pending or issued patent is licensed, GMP and LH would be entitled to a share of any proceeds Mount Sinai School of Medicine receives from the license.

\section{References}

1. Alzheimer's Association. 2008 Alzheimer's Disease Facts and Figures. Alzheimers Dement. 2008;4:110-133.

2. Cummings JL. Treatment of Alzheimer's disease: Current and future therapeutic approaches. Rev Neurol Dis. 2004;1:60-69.

3. Sano M. Current concepts in the prevention of Alzheimer's disease. CNS Spectr. 2003;8:846-853.

4. Religa D, Winblad B. Therapeutic strategies for Alzheimer's disease based on new molecular mechanisms. Acta Neurobiol Exp. 2003;63(4): 393-396.

5. Pangalos MN, Jacobsen SJ, Reinhart PH. Disease modifying strategies for the treatment of Alzheimer's disease targeted at modulating levels of beta-amyloid peptide. Biochem Soc Trans. 2005;33(Pt 4):553-558.

6. Duyckaerts C, Delatour B, Potier MC. Classification and basic pathology of Alzheimer's disease. Acta Neuropathol. 2009;11:5-36.

7. Davies P, Koppel J. Mechanism-based treatment for Alzheimer's disease. Dialogues Clin Neurosci. 2009;11:159-169.

8. Kukar T, Murphy MP, Eriksen JL, et al. Diverse compounds mimic Alzheimer's disease-causing mutations by augmenting A $\beta 42$ production. Nat Med. 2005;11:545-550.

9. Golde TE, Eckman CB, Younkin SG. Biochemical detection of A $\beta$ isoforms: Implications for pathogenesis, diagnosis, and treatment of Alzheimer's disease. Biochim Biophys Acta. 2000;1502:172-187.

10. Scheuner D, Eckman C, Jensen M, et al. Secreted amyloid beta-protein similar to that in the senile plaques of Alzheimer's disease is increased in vivo by the presenilin 1 and 2 and APP mutations linked to familial Alzheimer's disease. Nat Med. 1996;2:864-870.

11. Duff K, Eckman C, Zehr C, et al. Increased amyloid-beta42(43) in brains of mice expressing mutant presenilin 1. Nature. 1996;383:710-713.

12. Cleary JP, Walh DM, Hofmeister JJ, et al. Natural oligomers of the amyloid-beta protein specifically disrupt cognitive function. Nat Neurosci. 2005;8:79-84.

13. Lesne S, Koh MT, Kotilinek L, et al. A specific amyloid-beta protein assembly in the brain impairs memory. Nature. 2006;440(7082): $352-357$.

14. Jacobsen JS, Wu CC, Redwine JM, et al. Early-onset behavioral and synaptic deficits in a mouse model of Alzheimer's disease. Proc Natl Acad Sci U S A. 2006;103:5161-5166.
15. Walsh DM, Klyubin I, Fadeeva JV, et al. Naturally secreted oligomers of amyloid beta protein potently inhibit hippocampal long-term potentiation in vivo. Nature. 2002;416:535-539.

16. Klyubin I, Walsh DM, Lemere CA, et al. Amyloid beta protein immunotherapy neutralizes abeta oligomers that disrupt synaptic plasticity in vivo. Nat Med. 2005;11:556-561.

17. Hardy J, Selkoe DJ. The amyloid hypothesis of Alzheimer's disease: Progress and problems on the road to therapeutics. Science. 2002; 297(5580):353-356.

18. Goedert M. Tau protein and the neurofibrillary pathology of Alzheimer's disease. Ann NY Acad Sci. 1996;77:121-131.

19. Sahara N, Maeda S, Takashima A. Tau oligomerization: A role for tau aggregation intermediates linked to neurodegeneration. Curr Alzheimer Res. 2008;5:591-598.

20. Santacruz K, Lewis J, Spires T, et al. Tau suppression in a neurodegenerative mouse model improves memory function. Science. 2005; 309(5733):476-481.

21. Berger Z, Roder H, Hanna A, et al. Accumulation of pathological tau species and memory loss in a conditional model of tauopathy. $J \mathrm{Neu}$ rosci. 2007;27:3650-3652.

22. Sorrentino G, Bonavita V. Neurodegeneration and Alzheimer's disease: The lesson from tauopathies. Neurol Sci. 2007;28:63-71.

23. Ono K, Condron MM, Ho L, et al. Effects of grape seed-derived polyphenols on amyloid beta-protein self-assembly and cyotoxicity. $J$ Biol Chem. 2008;283:32176-32187.

24. Ho L, Yemul S, Wang J, Pasinetti GM. Grape seed polyphenolic extract as a potential novel therapeutic agent in tauopathies. J Alzheimers Dis. 2009; 16:433-439.

25. Wang J, Ho L, Zhao W, et al. Grape-derived polyphenolics prevent abeta oligomerization and attenuate cognitive deterioration in a mouse model of Alzheimer's disease. J Neurosci. 2008;28:6388-6392.

26. Song X, Siriwardhana N, Rathore K, Lin D, Wang HC. Grape seed proanthocyanidin suppression of breast cell carcinogenesis induced by chronic exposure to combined 4-(methylnitorsamino)-1-(3-pyridyl)-1butanone and benzo[a]pyrene. Mol Carcinog. 2010;49:450-463.

27. Sivaprakasapillai B, Edirisinghe I, Randolph J, Steinberg F, Kappagoda T. Effect of grape seed extract on blood pressure in subjects with the metabolic syndrome. Metabolism. 2009;58:1743-1746.

28. Montagut G, Onnockx S, Vaque M, et al. Oligomers of a grape-seed procyanidin extract activate the insulin receptor and key targets of the insulin signaling pathway differently from insulin. $J$ Nutr Biochem. 2010;2:476-481.

29. Karaaslan O, Ulusoy MG, Kankaya Y, et al. Protective effect of grape seed extract against ischaemia/reperfusion injury in a rat epigastic flap model. J Plast Reconstr Aesthet Surg. 2010;63:705-710.

30. Khanna S, Venojarvi M, Roy S, et al. Dermal wound healing properties of redox-active grape seed proanthocyanidins. Free Radic Biol Med. 2002;33:1089-1096.

31. Li X, Xhao Y, Gao H, et al. Grape seed proanthocyanidins ameliorate diabetic nephropathy via modulation of levels of AGE, RAGE and CTGH. Nephron Exp Nephrol. 2009;111:e31-e41.

32. Bagchi D, Bagchi M, Stohs SJ, et al. Free radicals and grape seed proanthocyanidin extract: Importance in human health and disease prevention. Toxicology. 2000;148:187-197.

33. Montagut G, Blade C, Blay M, et al. Effects of a grape seed procyanidin extract (GSPE) on insulin resistance. J Nutr Biochem. Dec 3, 2009. [Epub ahead of print].

34. Wang YJ, Thomas $\mathrm{P}$, Zhong JH, et al. Consumption of grape seed extract prevents amyloid-beta deposition and attenuates inflammation in brain of an Alzheimer's disease mouse. Neurotox Res. 2009;15:3-14.

35. Singleton VL, Orthofer R, Lamuela-Raventos RM. Analysis of total phenols and other oxidation substrates and antioxidants by means of Folin-Ciocalteu reagent. Methods Enzymol. 1999;299:152-178.

36. Ferruzzi MG, Lobo JK, Janle EM, et al. Bioavailability of gallic acid and catechins from grape seed polyphenol extract is improved by repeated dosing in rats: Implications for treatment in Alzheimer's disease. J Alzheimers Dis. 2009;1:113-124. 
37. Hsiao K, Chapman P, Nilsen S, et al. Correlative memory deficits, abeta elevation, and amyloid plaques in transgenic mice. Science. 1996;274: 99-103.

38. Barghorn S, Davies P, Mandelkow E. Tau paired helical filaments from Alzheimer's disease brain and assembled in vitro are based on betastructure in the core domain. Biochemistry. 2004;43:1694-1703.

39. Kajava AV, Baxa U, Wickner RB, Steven AC. A model for Ure2p prion filaments and other amyloids: The parallel superpleated beta-structure. Proc Natl Acad Sci U S A. 2004;101:7885-7890.

40. Lindwall $\mathrm{G}$, Cole RD. The purification of tau protein and the occurrence of two phosphorylation states of tau in brain. J Biol Chem. 1984; 259:12241-12245.

41. Schweers O, Schonbrunn-Hanebeck E, Marx A, Mandelkow E. Structural studies of tau protein and Alzheimer paired helical filaments show no evidence for beta-structure. J Biol Chem. 1994;269:24290-24297.

42. von Bergen M, Barghorn S, Biernat J, Mandelkow EM, Mandelkow E. Tau aggregation is driven by a transition from random coil to beta sheet structure. Biochim Biophys Acta. 2005;1739:158-166.
43. Berriman J, Serpell LC, Oberg KA, Fink AL, Goedert M, Crowther RA. Tau filaments from human brain and from in vitro assembly of recombinant protein show cross-beta structure. Proc Natl Acad Sci U S A. 2003;100:9034-9038.

44. Giannetti AM, Lindwall G, Chau MF, Radeke MJ, Feinstein SC, Kojlstaedt LA. Fibers of tau filaments, but not full length tau, exhibit a cross beta-structure: Implications for the formation of paired helical filaments. Protein Sci. 2000;9:2424-2435.

45. Sadqi M, Hernandez F, Pan U, et al. Alpha-helix structure in Alzheimer's disease aggregates of tau-protein. Biochemistry. 2002;41:7150-7155.

46. Goux WJ, Kopplin L, Nguyen AD, et al. The formation of straight and twisted filaments from short tau peptides. J Biol Chem. 2004;279 26868-26875.

47. Bentivegna SS, Whitney KM. Subchronic 3-month oral toxicity study of grape seed and grape skin extracts. Food Chem Toxicol. 2002;40 1731-1743.
Nutrition and Dietary Supplements

\section{Publish your work in this journal}

Nutrition and Dietary Supplements is an international, peer-reviewed open access journal focusing on research into nutritional requirements in health and disease, impact on metabolism and the identification and optimal use of dietary strategies and supplements necessary for normal growth and development. The journal welcomes papers covering

\section{Dovepress}

original research, basic science, clinical \& epidemiological studies, reviews and evaluations, guidelines, expert opinion and commentary, case reports and extended reports. The manuscript management system is completely online and includes a very quick and fair peer-review system, which is all easy to use.

Submit your manuscript here: http://www.dovepress.com/nutrition-and-dietary-supplements-journal 